\section{Eisen im Blick bei Hypothyreose}

Es scheint ein Zusammenhang zwischen einer Hypothyreose und einer Eisenmangelanämie zu bestehen. Dies haben Ärzte der Universität Bushehr im Iran in einer randomisierten Doppelblindstudie untersucht. 60 Patienten mit Eisenmangelanämie und subklinischer Hypothyreose wurden dazu entweder mit Eisensalz plus Placebo, mit Levothyroxin plus Placebo oder mit Eisensalz plus Levothyroxin behandelt. Nach dreimonatiger Behandlung war nur bei den doppelt substituierten Patienten ein signifikanter Anstieg von Hämoglobin und Ferritin messbar, und zwar um 1,17 g/dl bzw. um 10,78 $\mu \mathrm{g} / \mathrm{l}$.

Ravanbod M et al, The American Journal of Medicine 2013, 126: 420-424

\section{Masern halten sich hartnäckig}

Die Masern-Elimination ist noch in mehreren Ländern Europas in weiter Ferne. So wurden in den 29 Ländern der WHORegion Europa 2012 insgesamt 8032 Erkrankungen registriert, meldet das ECDC. In Deutschland war die Situation 2012 mit nur 168 gemeldeten Masernfällen relativ ruhig. 2013 wurden bis 8. April $37 \mathrm{Er}-$ krankungen gemeldet. Damit wurde auch hierzulande das Eliminations-Ziel mit weniger als einer Erkrankung pro einer Million Einwohner verfehlt.

Ärzte Zeitung, 21.4.2013

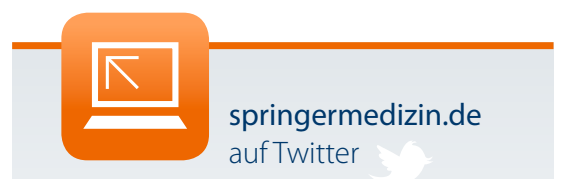

Auch im Frühling heißt

es wieder:

"Lauschen" Sie unserem

Zwitschern und werden Sie zum

"Follower".

-twitter.com/springermedizin

Heilsame Nitrate

\title{
Blutdrucksenker Rote Bete
}

Forscher fanden heraus, dass die Nitrate in Roter Bete den Blutdruck nicht nur bei gesunden Menschen, sondern auch bei Hypertonikern senken - bei letzteren offenbar sogar besonders gut.

In Roter Bete stecken anscheinend vasoaktive Kräfte. Ein Forscherteam um Dr. Amrita Ahluwalia, London School of Medicine and Dentristy, fand jetzt heraus, dass $250 \mathrm{ml}$ Rote-Bete-Saft am Tag den Blutdruck bei Hypertonikern senkt. Die hohe Konzentration an anorganischem Nitrat (NO3-) in der Roten Bete scheint dafür verantwortlich zu sein.

Die Forscher haben acht Frauen und sieben Männer mit Bluthochdruck ersten Grades randomisiert zwei Gruppen zugeteilt: Die einen tranken $250 \mathrm{ml}$ Rote BeteSaft am Tag, die anderen die gleiche Menge an Wasser.

Die im Saft enthaltene relativ geringe Nitrat-Dosis reichte aus, um sowohl den systolischen als auch den diastolischen
Blutdruck der Hypertoniker signifikant zu senken.

Im Mittel wurde der systolische Blutdruck drei bis sechs Stunden nach dem Rote Bete-Trank um 11,2 mmHg gesenkt, im Vergleich zu 0,7 $\mathrm{mmHg}$ bei den Probanden, die Wasser tranken. Auch der diastolische Blutdruck war vier bis sechs Stunden nach Zufuhr des Rote-Bete-Safts signifikant niedriger als der von „Wasser-Trinkern“, und zwar um 9,6 mmHg.

Dabei korrelierte die Höhe der Blutdrucksenkung mit der Höhe der NitritSpiegel im Blut.

Die Nitrate scheinen bei Hypertonikern sogar noch effektiver zu wirken als bei gesunden Menschen. So zeigte der direkte Vergleich im Tiermodell eine deutlich stärkere Blutdrucksenkung bei hypertensiven Ratten als bei normotensiven Tieren.

Ahluwalia A,Ghosh S, Kapil V, et al., Hypertension 2013 DOI:10.1161/HYPERTENSIONAHA.111.00933

\section{Zerbrechliche alte Schultern}

\section{Beim Reponieren bricht leicht der Arm}

Erstmalige Schulterluxationen sollten bei älteren Patienten besser nicht notfallmäßig reponiert werden. Häufig liegen Begleitverletzungen vor, die das Risiko einer iatrogenen Fraktur erhöhen.

Um iatrogene Oberarmfrakturen zu vermeiden, empfehlen die Forscher um Ehud Atoun vom Royal Berkshire Hospital im britischen Reading, Patienten über 40 ausschließlich in Allgemeinanästhesie und unter vollständiger Muskelrelaxation zu reponieren.

An ihrer retrospektiven Studie hatten 92 erstmals schulterluxierte Patienten im Alter von über 40 Jahren teilgenommen.
Wie die Forscher berichten, lag die Rate der dislozierten Humerushalsfrakturen bei 5,4\%. In allen diesen Fällen hatte man eine initial vorliegende Fraktur des Tuberculum majus im Röntgenbild übersehen. Damit hatte die Reposition bei jedem vierten Patienten mit einer solchen vorbestehenden Verletzung zu einer zusätzlichen Fraktur geführt. Welche Repositionsmanöver zum Einsatz gekommen waren, ließ sich allerdings nicht mehr feststellen.

Alle betroffenen Patienten mussten anschließend operiert werden und litten nach der Operation unter Komplikationen.

Atoun Eet al, J Orthop Trauma 2013; 27: 190-195 